\title{
Invertibility of Matrix Wiener-Hopf plus Hankel Operators with Symbols Producing a Positive Numerical Range
}

\author{
L. P. Castro and A. S. Silva
}

\begin{abstract}
We characterize left, right and both-sided invertibility of matrix WienerHopf plus Hankel operators with possibly different Fourier symbols in the Wiener subclass of the almost periodic algebra. This is done when a certain almost periodic matrix-valued function (constructed from the initial Fourier symbols of the Hankel and Wiener-Hopf operators) admits a numerical range bounded away from zero. The invertibility characterization is based on the value of a certain mean motion. At the end, an example of a concrete Wiener-Hopf plus Hankel operator is studied in view of the illustration of the proposed theory.
\end{abstract}

Keywords. Wiener-Hopf operator, Hankel operator, almost periodic function, invertibility

Mathematics Subject Classification (2000). Primary 47B35, secondary 47A05, 47A12, 47A20, 42A75

\section{Introduction}

Motivated by the needs of different kinds of applications, there is a growing interest in the study of invertibility properties of the so-called Wiener-Hopf plus Hankel operators (cf., e.g., $[4,7-10,12,17,19,21,22,24,25])$. In fact, these operators occur in a natural manner in many applications. E.g., in the analysis of problems of wave diffraction by wedges (cf. $[8,9,25])$ this is particularly evident due to the use of some symmetrization techniques which generate sums of Hankel and Wiener-Hopf operators. Therefore, an eventual additional knowledge about the invertibility characteristics of these operators is welcome for both theoretical and practical reasons.

L. P. Castro: Department of Mathematics, University of Aveiro, 3810-193 Aveiro, Portugal; castro@ua.pt

A. S. Silva: Department of Mathematics, University of Aveiro, 3810-193 Aveiro, Portugal; anabela.silva@ua.pt

Sponsored by Fundação para a Ciência e a Tecnologia (Portugal) under grant number $\mathrm{SFRH} / \mathrm{BD} / 38698 / 2007$. 
In the present paper, we will consider matrix Wiener-Hopf plus Hankel operators of the form

$$
W_{\Phi_{1}}+H_{\Phi_{2}}:\left[L_{+}^{2}(\mathbb{R})\right]^{n} \rightarrow\left[L^{2}\left(\mathbb{R}_{+}\right)\right]^{n},
$$

with $W_{\Phi_{1}}$ and $H_{\Phi_{2}}$ being matrix Wiener-Hopf and Hankel operators defined by

$$
W_{\Phi_{1}}=r_{+} \mathcal{F}^{-1} \Phi_{1} \cdot \mathcal{F} \quad \text { and } \quad H_{\Phi_{2}}=r_{+} \mathcal{F}^{-1} \Phi_{2} \cdot \mathcal{F} J
$$

respectively. We use $\left[L_{+}^{2}(\mathbb{R})\right]^{n}$ to denote the subspace of $\left[L^{2}(\mathbb{R})\right]^{n}$ formed by all the vector functions supported on the closure of $\mathbb{R}_{+}=(0,+\infty), r_{+}$represents the operator of restriction from $\left[L^{2}(\mathbb{R})\right]^{n}$ onto $\left[L^{2}\left(\mathbb{R}_{+}\right)\right]^{n}, \mathcal{F}$ denotes the Fourier transformation, $J$ is the reflection operator given by the rule $J \varphi(x)=$ $\widetilde{\varphi}(x)=\varphi(-x), x \in \mathbb{R}$, and $\Phi_{1}$ and $\Phi_{2}$ are $n \times n$ matrix functions with elements belonging to the so-called $A P W$ algebra. For defining this algebra, let us first consider the algebra of almost periodic functions, usually denoted by $A P$, i.e., the smallest closed subalgebra of $L^{\infty}(\mathbb{R})$ that contains all the functions $e_{\lambda}(\lambda \in \mathbb{R})$, where

$$
e_{\lambda}(x)=e^{i \lambda x}, x \in \mathbb{R} .
$$

$A P W$ is the subclass of all functions $\varphi \in A P$ which can be written in the form of an absolutely convergent series, i.e.,

$$
A P W:=\left\{\varphi=\sum_{j} \varphi_{j} e_{\lambda_{j}}: \varphi_{j} \in \mathbb{C}, \lambda_{j} \in \mathbb{R}, \sum_{j}\left|\varphi_{j}\right|<\infty\right\}
$$

$A P W$ becomes a Banach algebra with respect to pointwise addition and multiplication when endowed with the norm $\|\varphi\|_{A P W}:=\sum_{j}\left|\varphi_{j}\right|$ (with $\varphi_{j}$ in the sense of (1.2)).

We will use the notation $\mathcal{G} B$ for the group of all invertible elements of a Banach algebra $B$. Applying a similar result of Bohr's Theorem for AP functions, it holds that for each $\phi \in \mathcal{G} A P W$ there exists a real number $\kappa(\phi)$ and a function $\psi \in A P W$ such that

$$
\phi(x)=e^{i \kappa(\phi) x} e^{\psi(x)},
$$

for all $x \in \mathbb{R}$ (cf. Theorem 8.11 in [3]). The number $\kappa(\phi)$ is uniquely determined, and is called the mean motion of $\phi$.

We would like to clarify that in opposition to the Wiener-Hopf plus Hankel case, the properties of Wiener-Hopf operators with almost periodic symbols are already well developed (cf., e.g., $[3,11]$ ). In addition, here we are considering the possibility of $\Phi_{1} \neq \Phi_{2}$ (see (1.1)) in contrast to some previous works that study regularity properties of Wiener-Hopf plus Hankel operators only in the case of $\Phi_{1}=\Phi_{2} ;$ cf. $[4,5,20-22]$. 


\section{Operator relations for Wiener-Hopf plus Hankel operators with essentially bounded Fourier symbols}

The main purpose of this section is to present an explicit operator relation between the above defined Wiener-Hopf plus Hankel operator $W_{\Phi_{1}}+H_{\Phi_{2}}$ and a new Wiener-Hopf operator. This will be done in the form of a so-called delta relation after extension [6].

For such a purpose, let us first recall some different types of relations between bounded linear operators. Consider two bounded linear operators $T: X_{1} \rightarrow X_{2}$ and $S: Y_{1} \rightarrow Y_{2}$, acting between Banach spaces. The operators $T$ and $S$ are said to be equivalent, and we will denote this by $T \sim S$, if there are two boundedly invertible linear operators, $E: Y_{2} \rightarrow X_{2}$ and $F: X_{1} \rightarrow Y_{1}$, such that

$$
T=E S F \text {. }
$$

It directly follows from (2.1) that if two operators are equivalent, then they belong to the same invertibility class [6,23]. More precisely, one of these operators is invertible, left invertible, right invertible or only generalized invertible, if and only if the other operator enjoys the same property.

The so-called $\Delta$-relation after extension was introduced in [6] for bounded linear operators acting between Banach spaces, e.g. $T: X_{1} \rightarrow X_{2}$ and $S$ : $Y_{1} \rightarrow Y_{2}$. We say that $T$ is $\Delta$-related after extension to $S$ if there is a bounded linear operator acting between Banach spaces $T_{\Delta}: X_{1 \Delta} \rightarrow X_{2 \Delta}$ and invertible bounded linear operators $E$ and $F$ such that

$$
\left[\begin{array}{cc}
T & 0 \\
0 & T_{\Delta}
\end{array}\right]=E\left[\begin{array}{cc}
S & 0 \\
0 & I_{Z}
\end{array}\right] F,
$$

where $Z$ is an additional Banach space and $I_{Z}$ represents the identity operator in $Z$. In the particular case when $T_{\Delta}: X_{1 \Delta} \rightarrow X_{2 \Delta}=X_{1 \Delta}$ is the identity operator, we say that the operators $T$ and $S$ are equivalent after extension. It follows from (2.2) that if we have $T$ being $\Delta$-related after extension to $S$, then the transfer of invertibility properties can only be guaranteed in one direction, that is, from operator $S$ to operator $T$.

We are now in conditions to present the above announced result which is based on the famous Gohberg-Krupnik-Litvinchuk identity (see [13-16,18], for instance).

Theorem 2.1. Let $\Phi_{1}, \Phi_{2} \in \mathcal{G}\left[L^{\infty}(\mathbb{R})\right]^{n \times n}$. Then the matrix Wiener-Hopf plus Hankel operator $W_{\Phi_{1}}+H_{\Phi_{2}}:\left[L_{+}^{2}(\mathbb{R})\right]^{n} \rightarrow\left[L^{2}\left(\mathbb{R}_{+}\right)\right]^{n}$ is $\Delta$-related after extension to the Wiener-Hopf operator $W_{\Psi}:\left[L_{+}^{2}(\mathbb{R})\right]^{2 n} \rightarrow\left[L^{2}\left(\mathbb{R}_{+}\right)\right]^{2 n}$ with Fourier symbol

$$
\Psi=\left[\begin{array}{cc}
\Phi_{1}-\widetilde{\Phi_{2} \widetilde{\Phi_{1}^{-1}} \widetilde{\Phi_{2}}} & -\widetilde{\Phi_{2} \widetilde{\Phi_{1}^{-1}}} \\
\widetilde{\Phi_{1}^{-1}} \widetilde{\Phi_{2}} & \widetilde{\Phi_{1}^{-1}}
\end{array}\right] .
$$


Proof. Let us start by extending $W_{\Phi_{1}}+H_{\Phi_{2}}$ on the left by the zero extension operator $\ell_{0}:\left[L^{2}\left(\mathbb{R}_{+}\right)\right]^{n} \rightarrow\left[L_{+}^{2}(\mathbb{R})\right]^{n}$, which leads to

$$
W_{\Phi_{1}}+H_{\Phi_{2}} \sim \ell_{0}\left(W_{\Phi_{1}}+H_{\Phi_{2}}\right):\left[L_{+}^{2}(\mathbb{R})\right]^{n} \rightarrow\left[L_{+}^{2}(\mathbb{R})\right]^{n} .
$$

Using the notation $P_{+}=\ell_{0} r_{+}$and $P_{-}=I_{\left[L^{2}(\mathbb{R})\right]^{n}}-P_{+}$, we will now extend

$$
\ell_{0}\left(W_{\Phi_{1}}+H_{\Phi_{2}}\right)=P_{+} \mathcal{F}^{-1}\left(\Phi_{1} \cdot+\Phi_{2} \cdot J\right) \mathcal{F}_{\mid P_{+}\left[L^{2}(\mathbb{R})\right]^{n}}
$$

to the full $\left[L^{2}(\mathbb{R})\right]^{n}$ space by using the identity in $\left[L_{-}^{2}(\mathbb{R})\right]^{n}$. Next we will extend the obtained operator to $\left[L^{2}(\mathbb{R})\right]^{2 n}$ with the help of the auxiliary paired operator

$$
\mathcal{T}=\mathcal{F}^{-1}\left(\Phi_{1} \cdot-\Phi_{2} \cdot J\right) \mathcal{F} P_{+}+P_{-}:\left[L^{2}(\mathbb{R})\right]^{n} \rightarrow\left[L^{2}(\mathbb{R})\right]^{n}
$$

Altogether, we have

$$
\left[\begin{array}{cc|c}
\ell_{0}\left(W_{\Phi_{1}}+H_{\Phi_{2}}\right) & 0 & 0 \\
0 & I_{P_{-}\left[L^{2}(\mathbb{R})\right]^{n}} & 0 \\
\hline 0 & 0 & \mathcal{T}
\end{array}\right]=E_{1} \mathcal{W}_{1} F_{1}
$$

with

$$
\begin{aligned}
& E_{1}=\frac{1}{2}\left[\begin{array}{cc}
I_{\left[L^{2}(\mathbb{R})\right]^{n}} & J \\
I_{\left[L^{2}(\mathbb{R})\right]^{n}} & -J
\end{array}\right] \\
& F_{1}=\left[\begin{array}{cc}
I_{\left[L^{2}(\mathbb{R})\right]^{n}} & I_{\left[L^{2}(\mathbb{R})\right]^{n}} \\
J & -J
\end{array}\right]\left[\begin{array}{cc}
I_{\left[L^{2}(\mathbb{R})\right]^{n}}-P_{-} \mathcal{F}^{-1}\left(\Phi_{1} \cdot+\Phi_{2} \cdot J\right) \mathcal{F} P_{+} & 0 \\
0 & I_{\left[L^{2}(\mathbb{R})\right]^{n}}
\end{array}\right] \\
& \mathcal{W}_{1}=\left[\begin{array}{ll}
\mathcal{F}^{-1} \Phi_{1} \cdot \mathcal{F} & 0 \\
\mathcal{F}^{-1} \widetilde{\Phi_{2}} \cdot \mathcal{F} & 1
\end{array}\right] P_{+}+\left[\begin{array}{ll}
1 & \mathcal{F}^{-1} \Phi_{2} \cdot \mathcal{F} \\
0 & \mathcal{F}^{-1} \widetilde{\Phi_{1}} \cdot \mathcal{F}
\end{array}\right] P_{-} \\
& =\left[\begin{array}{cc}
1 & \mathcal{F}^{-1} \Phi_{2} \cdot \mathcal{F} \\
0 & \mathcal{F}^{-1} \widetilde{\Phi_{1}} \cdot \mathcal{F}
\end{array}\right]\left(\mathcal{F}^{-1} \Psi \cdot \mathcal{F} P_{+}+P_{-}\right) \\
& =\left[\begin{array}{cc}
1 & \mathcal{F}^{-1} \Phi_{2} \cdot \mathcal{F} \\
0 & \mathcal{F}^{-1} \widetilde{\Phi_{1}} \cdot \mathcal{F}
\end{array}\right]\left(P_{+} \mathcal{F}^{-1} \Psi \cdot \mathcal{F} P_{+}+P_{-}\right)\left(I_{\left[L^{2}(\mathbb{R})\right]^{2 n}}+P_{-} \mathcal{F}^{-1} \Psi \cdot \mathcal{F} P_{+}\right),
\end{aligned}
$$

where in the last definition of operator $\mathcal{W}_{1}$ we are using $P_{ \pm}$defined in $\left[L^{2}(\mathbb{R})\right]^{2 n}$ and $\Psi$ is the same as defined in (2.3). Note that the paired operator

$$
I_{\left[L^{2}(\mathbb{R})\right]^{2 n}}+P_{-} \mathcal{F}^{-1} \Psi \cdot \mathcal{F} P_{+}:\left[L^{2}(\mathbb{R})\right]^{2 n} \rightarrow\left[L^{2}(\mathbb{R})\right]^{2 n}
$$

used above is an invertible operator with inverse given by

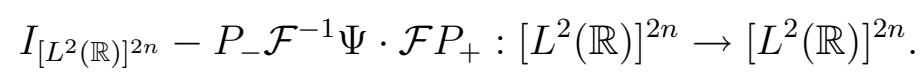


Therefore, we have just explicitly demonstrated that $W_{\Phi_{1}}+H_{\Phi_{2}}$ is $\Delta$-related after extension to the Wiener-Hopf operator

$$
W_{\Psi}=r_{+} \mathcal{F}^{-1} \Psi \cdot \mathcal{F}:\left[L_{+}^{2}(\mathbb{R})\right]^{2 n} \rightarrow\left[L^{2}\left(\mathbb{R}_{+}\right)\right]^{2 n},
$$

and this concludes the proof.

Corollary 2.2. Let $\Phi_{1}, \Phi_{2} \in \mathcal{G}\left[L^{\infty}(\mathbb{R})\right]^{n \times n}$. If the Wiener-Hopf operator $W_{\Psi}$ : $\left[L_{+}^{2}(\mathbb{R})\right]^{2 n} \rightarrow\left[L^{2}\left(\mathbb{R}_{+}\right)\right]^{2 n}$ is Fredholm or (left/right/both-sided) invertible, then the Wiener-Hopf plus Hankel operator $W_{\Phi_{1}}+H_{\Phi_{2}}:\left[L_{+}^{2}(\mathbb{R})\right]^{n} \rightarrow\left[L^{2}\left(\mathbb{R}_{+}\right)\right]^{n}$ has the same corresponding property.

Proof. Due to the $\Delta$-relation after extension between the two operators presented in Theorem 2.1, we derive that:

(i) $\operatorname{im}\left[\begin{array}{cc}W_{\Phi_{1}}+H_{\Phi_{2}} & 0 \\ 0 & \mathcal{T}\end{array}\right]$ is closed if and only if $\operatorname{im} W_{\Psi}$ is closed;

(ii) $\left(\left[L^{2}\left(\mathbb{R}_{+}\right)\right]^{n} \times\left[L^{2}(\mathbb{R})\right]^{n}\right) \backslash \overline{\operatorname{im}\left[\begin{array}{cc}W_{\Phi_{1}}+H_{\Phi_{2}} & 0 \\ 0 & \mathcal{T}\end{array}\right]} \simeq\left[L^{2}\left(\mathbb{R}_{+}\right)\right]^{2 n} \backslash \overline{\operatorname{im} W_{\Psi}}$

(iii) $\operatorname{ker}\left[\begin{array}{cc}W_{\Phi_{1}}+H_{\Phi_{2}} & 0 \\ 0 & \mathcal{T}\end{array}\right] \simeq \operatorname{ker} W_{\Psi}$.

Then, these properties (i)-(iii) are enough to conclude the desired statement, taking into consideration the definitions of Fredholm property and (lateral/both-sided) invertibility.

\section{An invertibility characterization based on a mean motion and a numerical range}

We recall that the numerical range of a complex matrix $\Theta \in \mathbb{C}^{m \times m}$ is defined by

$$
\mathcal{H}(\Theta)=\left\{(\Theta \eta, \eta): \eta \in \mathbb{C}^{m},\|\eta\|=1\right\} .
$$

If $\Phi \in[A P W]^{m \times m}$, then (due to the definition of $A P W$ ) we also have that $\mathcal{H}(\Phi(x))$ is well-defined for all $x \in \mathbb{R}$. In this way, the numerical range of $\Phi$ is said to be bounded away from zero if $\inf _{x \in \mathbb{R}} \operatorname{dist}(\mathcal{H}(\Phi(x)), 0)>0$ or, equivalently, if there is an $\varepsilon>0$ such that

$$
|(\Phi(x) \eta, \eta)| \geq \varepsilon\|\eta\|^{2} \quad \text { for all } x \in \mathbb{R} \text { and all } \eta \in \mathbb{C}^{m} .
$$

Consider $\Phi \in[A P W]^{m \times m}$ and $\eta \in \mathbb{C}^{m} \backslash\{0\}$. If the numerical range of $\Phi$ is bounded away from zero, then the function $(\Phi \eta, \eta)$ given by

$$
(\Phi \eta, \eta)(x)=(\Phi(x) \eta, \eta), \quad x \in \mathbb{R}
$$


is invertible in $A P W$. Thus, the mean motion of $(\Phi \eta, \eta)$, denoted by $\kappa((\Phi \eta, \eta))$, is well-defined for all $\eta \in \mathbb{C}^{m} \backslash\{0\}$. Moreover, due to a theorem by Babadzhanyan and Rabinovich (see Theorem 9.9 in [3], and cf. also [1], [2]), we have that $\kappa((\Phi \eta, \eta))$ is independent of $\eta \in \mathbb{C}^{m} \backslash\{0\}$.

Theorem 3.1. Let us consider $\Phi_{1}, \Phi_{2} \in \mathcal{G}[A P W]^{n \times n}$ such that the numerical range of $\Psi$ (defined in (2.3)) is bounded away from zero. We have the following characterization of the invertibility properties of the operator $W_{\Phi_{1}}+H_{\Phi_{2}}$ :

(a) If $\kappa((\Psi \eta, \eta))=0$, then $W_{\Phi_{1}}+H_{\Phi_{2}}$ is invertible.

(b) If $\kappa((\Psi \eta, \eta))>0$, then $W_{\Phi_{1}}+H_{\Phi_{2}}$ is left invertible.

(c) If $\kappa((\Psi \eta, \eta))<0$, then $W_{\Phi_{1}}+H_{\Phi_{2}}$ is right invertible.

Proof. The assertion is now a consequence of the $\Delta$-relation after extension presented in the last section, and of the corresponding result for Wiener-Hopf operators (cf. Corollary 9.10 in [3]). Indeed, first, the hypotheses in (a), (b), and (c) give us (from [3, Corollary 9.10]) the invertibility, left invertibility, and right invertibility of $W_{\Psi}$, respectively. Then, by using Corollary 2.2, these three cases lead us to the final conclusion about the Wiener-Hopf plus Hankel operator.

It is clear that the condition on the numerical range of $\Psi$ - to be bounded away from zero - is fundamental in the last result. In view of this, it is also clear that not all Fourier symbol matrix functions $\Phi_{1}$ and $\Phi_{2}$ in $\mathcal{G}[A P W]^{n \times n}$ yield such a property for the corresponding $\Psi$ matrix function. For instance,

$$
\Phi_{1}(x)=\Phi_{2}(x)=\left[\begin{array}{cc}
2 e^{e^{i x}} & e^{e^{i x}} \\
e^{-e^{i x}} & e^{-e^{i x}}
\end{array}\right], \quad x \in \mathbb{R}
$$

is invertible in $[A P W]^{2 \times 2}$ but produces a matrix function $\Psi$ which does not have a numerical range bounded away from zero. In fact, for $\Phi_{1}$ and $\Phi_{2}$ in (3.1), if we take any $\eta=\left(\eta_{1}, \eta_{2}, 0,0\right)^{\top} \in \mathbb{C}^{4}$ such that $\|\eta\|=1$ then a direct computation yields $([\Psi(x)] \eta, \eta)=0$ for all $x \in \mathbb{R}$. Note that in such computation the identity $\Phi_{1}=\Phi_{2}$ plays an important role. Anyway, we would like to point out that such a particular case of equal Fourier symbols $\Phi_{1}=\Phi_{2}$ is also possible to consider by using matrices bounded away from zero, and even in a simpler way than in the present more complex case (cf. [20, Chapter 6]).

\section{Example}

To illustrate the previous theorem, we will present in this last section a concrete case of an invertible Wiener-Hopf plus Hankel operator $W_{\phi_{1}}+H_{\phi_{2}}$ with different $A P W$ Fourier symbols $\phi_{1}$ and $\phi_{2}$ :

$$
\phi_{1}(x)=2 e^{2 e^{-i x}} \text { and } \phi_{2}(x)=e^{e^{i x}+e^{-i x}}, \quad x \in \mathbb{R} .
$$


Note that $\phi_{1}$ and $\phi_{2}$ are invertible and $\phi_{1}^{-1}, \phi_{2}^{-2} \in A P W$. This yields that $\phi_{1}$, $\phi_{2} \in \mathcal{G} A P W$. In this case, for the element $\Psi$ from the last section, we have the particular form

$$
\Psi(x)=\left[\begin{array}{cc}
\frac{3}{2} e^{2 e^{-i x}} & -\frac{1}{2} e^{e^{-i x}}-e^{i x} \\
\frac{1}{2} e^{-e^{i x}}+e^{-i x} & \frac{1}{2} e^{-2 e^{i x}}
\end{array}\right], \quad x \in \mathbb{R} .
$$

Let us now analyze that the numerical range of $\Psi$ is bounded away from zero. Considering $\eta=\left(\eta_{1}, \eta_{2}\right)^{\top} \in \mathbb{C}^{2}$, such that $\|\eta\|=1$, it follows that

$$
\begin{aligned}
(\Psi(x) \eta, \eta) & =\left(\left[\begin{array}{c}
\frac{3}{2} \eta_{1} e^{2 e^{-i x}}-\frac{1}{2} \eta_{2} e^{e^{-i x}}-e^{i x} \\
\frac{1}{2} \eta_{1} e^{-e^{i x}+e^{-i x}}+\frac{1}{2} \eta_{2} e^{-2 e^{i x}}
\end{array}\right],\left[\begin{array}{l}
\eta_{1} \\
\eta_{2}
\end{array}\right]\right) \\
& =\frac{3}{2}\left|\eta_{1}\right|^{2} e^{2 e^{-i x}}+\frac{1}{2}\left|\eta_{2}\right|^{2} e^{-2 e^{i x}}+\Im m\left(\eta_{1} \overline{\eta_{2}}\right) i e^{-2 i \sin x},
\end{aligned}
$$

for all $x \in \mathbb{R}$. Therefore,

$$
\begin{aligned}
& \mathcal{H}(\Psi(x))= \\
& \left\{\frac{3}{2}\left|\eta_{1}\right|^{2} e^{2 e^{-i x}}+\frac{1}{2}\left|\eta_{2}\right|^{2} e^{-2 e^{i x}}+\Im m\left(\eta_{1} \overline{\eta_{2}}\right) i e^{-2 i \sin x}, \eta=\left[\begin{array}{l}
\eta_{1} \\
\eta_{2}
\end{array}\right] \in \mathbb{C}^{2},\|\eta\|=1\right\}
\end{aligned}
$$

for $x \in \mathbb{R}$. Then, we have that

$$
\operatorname{dist}(\mathcal{H}(\Psi(x)), 0)=\left[\left.\left|\frac{3}{2}\right| \eta_{1}\right|^{2} e^{2 e^{-i x}}+\frac{1}{2}\left|\eta_{2}\right|^{2} e^{-\left.2 e^{i x}\right|^{2}}+\left|\Im m\left(\eta_{1} \overline{\eta_{2}}\right) e^{-2 i \sin x}\right|^{2}\right]^{\frac{1}{2}}
$$

with $\eta=\left(\eta_{1}, \eta_{2}\right)^{\top} \in \mathbb{C}^{2}$ such that $\|\eta\|=1$.

Let $\|\eta\|=1$. It being clear that $\Im m\left(\eta_{1} \overline{\eta_{2}}\right) e^{-2 i \sin x}=0$ if and only if $\Im m\left(\eta_{1} \overline{\eta_{2}}\right)=0$, we will now verify that $\left.\left|\frac{3}{2}\right| \eta_{1}\right|^{2} e^{2 e^{-i x}}+\frac{1}{2}\left|\eta_{2}\right|^{2} e^{-2 e^{i x}} \mid \neq 0$ for all $x \in \mathbb{R}$. We have

$$
\begin{aligned}
\left.\left|\frac{3}{2}\right| \eta_{1}\right|^{2} e^{2 e^{-i x}}+\frac{1}{2}\left|\eta_{2}\right|^{2} e^{-2 e^{i x} \mid} & =\left.\left|\frac{3}{2}\right| \eta_{1}\right|^{2} e^{-2 i \sin x+2 \cos x}+\frac{1}{2}\left|\eta_{2}\right|^{2} e^{-2 i \sin x-2 \cos x} \mid \\
& =\left.\left|e^{-2 i \sin x}\right|\left|\frac{3}{2}\right| \eta_{1}\right|^{2} e^{2 \cos x}+\frac{1}{2}\left|\eta_{2}\right|^{2} e^{-2 \cos x} \mid \\
& =\left.\left|\frac{3}{2}\right| \eta_{1}\right|^{2} e^{2 \cos x}+\frac{1}{2}\left|\eta_{2}\right|^{2} e^{-2 \cos x} \mid>0 .
\end{aligned}
$$

Altogether, this leads to

$$
\inf _{x \in \mathbb{R}} \operatorname{dist}(\mathcal{H}(\Psi(x)), 0)>0,
$$

i.e., the numerical range of $\Psi$ is bounded away from zero. 
Now, to compute the corresponding mean motion, we start by considering $\eta=(1,0)^{\top}$. From $(4.1)$, it follows that $(\Psi(x) \eta, \eta)=\frac{3}{2} e^{2 e^{-i x}}, x \in \mathbb{R}$. Due to the fact that $e^{2 e^{-i x}} \in \mathcal{G} A P W$, from the analogue of Bohr's Theorem for $A P$ functions (cf. (1.3)), we have that

$$
k((\Psi \eta, \eta))=0
$$

for $\eta=(1,0)^{\top}$. From (4.2) and (4.3), and according to the Babadzhanyan and Rabinovich Theorem (mentioned above), it follows that $k((\Psi \eta, \eta))=0$ for all $\eta \in \mathbb{C}^{2} \backslash\{0\}$. Therefore, applying Theorem 3.1, we conclude that $W_{\phi_{1}}+H_{\phi_{2}}$ is an invertible operator.

Acknowledgment. This work was supported in part by Unidade de Investigação Matemática e Aplicações of Universidade de Aveiro through the Portuguese Science Foundation (FCT-Fundação para a Ciência e a Tecnologia).

\section{References}

[1] Babadzhanyan, R. G. and Rabinovich, V. S., A system of integral-difference equations on the half line (in Russian). Akad. Nauk Armyan. SSR Dokl. 81 (1985)(3), $107-111$.

[2] Babadzhanyan, R. G. and Rabinovich, V. S., Factorization of almost periodic operator functions (in Russian). In: Differential and Integral Equations and Complex Analysis. Elista: Kalmytsk. Gos. Univ. 1986, pp. 13 - 22.

[3] Böttcher, A., Karlovich, Yu. I. and Spitkovsky, I. M., Convolution Operators and Factorization of Almost Periodic Matrix Functions. Basel: Birkhäuser 2002.

[4] Bogveradze, G. and Castro, L. P., Wiener-Hopf plus Hankel operators on the real line with unitary and sectorial symbols. In: Operator Theory, Operator Algebras, and Applications. Contemp. Math. 414. Providence (RI): Amer. Math. Soc. 2006, pp. $77-85$.

[5] Bogveradze, G. and Castro, L. P., On the Fredholm index of matrix WienerHopf plus/minus Hankel operators with semi-almost periodic symbols. Oper. Theory Adv. Appl. 181 (2008), 143 - 158.

[6] Castro, L. P. and Speck, F.-O., Regularity properties and generalized inverses of delta-related operators. Z. Anal. Anwendungen 17 (1998), 577 - 598.

[7] Castro, L. P. and Speck, F.-O., Inversion of matrix convolution type operators with symmetry. Port. Math. (N.S.) 62 (2005)(2), $193-216$.

[8] Castro, L. P., Speck, F.-O. and Teixeira, F. S., Explicit solution of a DirichletNeumann wedge diffraction problem with a strip. J. Integral Eqs. Appl. 15 (2003), $359-383$. 
[9] Castro, L. P., Speck, F.-O. and Teixeira, F. S., On a class of wedge diffraction problems posted by Erhard Meister. Oper. Theory Adv. Appl. 147 (2004), $211-238$.

[10] Castro, L. P., Speck, F.-O. and Teixeira, F. S., A direct approach to convolution type operators with symmetry. Math. Nachr. 269/270 (2004), 73 - 85.

[11] Curto, R. E., Muhly, P. S. and Xia, J., Wiener-Hopf operators and generalized analytic functions. Integr. Eqs. Oper. Theory 8 (1985), $650-673$.

[12] Ehrhardt, T., Invertibility theory for Toeplitz plus Hankel operators and singular integral operators with flip. J. Funct. Anal. 208 (2004), 64-106.

[13] Gohberg, I. and Krupnik, N. Ya., One-dimensional singular integral operators with shift (in Russian). Izv. Akad. Nauk Arm. SSR, Matematika 8 (1973), $3-12$.

[14] Karapetiants, N. and Samko, S., Equations with Involutive Operators. Boston (MA): Birkhäuser 2001.

[15] Kravchenko, V. G. and Litvinchuk, G. S., Introduction to the Theory of Singular Integral Operators with Shift. Math. Appl. 289. Dordrecht: Kluwer 1994.

[16] Krupnik, N. Ya., Banach Algebras with Symbol and Singular Integral Operators. Basel: Birkhäuser 1987.

[17] Lebre, A. B., Meister, E. and Teixeira, F. S., Some results on the invertibility of Wiener-Hopf-Hankel operators. Z. Anal. Anwendungen 11 (1992), 57 - 76.

[18] Litvinchuk, G. S., Solvability Theory of Boundary Value Problems and Singular Integral Equations with Shift. Math. Appl. 523. Dordrecht: Kluwer 2000.

[19] Meister, E., Speck, F.-O. and Teixeira, F. S., Wiener-Hopf-Hankel operators for some wedge diffraction problems with mixed boundary conditions. J. Integral Eqs. Appl. 4 (1992), 229 - 255.

[20] Nolasco, A. P., Regularity Properties of Wiener-Hopf-Hankel Operators. PhD Thesis. Aveiro: University of Aveiro 2007.

[21] Nolasco, A. P. and Castro, L. P., Factorization theory for Wiener-Hopf plus Hankel operators with almost periodic symbols. In: Operator Theory, Operator Algebras, and Applications. Contemp. Math. 414. Providence (RI): Amer. Math. Soc. 2006, pp. $111-128$.

[22] Nolasco, A. P. and Castro, L. P., A Duduchava-Saginashvili's type theory for Wiener-Hopf plus Hankel operators. J. Math. Anal. Appl. 331 (2007), $329-341$.

[23] Speck, F.-O., General Wiener-Hopf Factorization Methods. London: Pitman 1985.

[24] Teixeira, F. S., On a class of Hankel operators: Fredholm properties and invertibility, Integr. Eqs. Oper. Theory 12 (1989), $592-613$.

[25] Teixeira, F. S., Diffraction by a rectangular wedge: Wiener-Hopf-Hankel formulation. Integr. Eqs. Oper. Theory 14 (1991), 436 - 454.

Received July 26, 2007; revised January 3, 2008 